\title{
Clinical analysis of acute cerebrovascular disease
}

\author{
Ronghua Zhao* \\ The Third Affiliated Hospital of Inner Mongolia Medical University, Baotou, Inner Mongolia, China
}

Received: January 23, 2017

DOI: $10.14725 /$ dcc.v4n1p14
Accepted: February 25, $2017 \quad$ Online Published: March 10, 2017

URL: http://dx.doi.org/10.14725/dcc.v4n1p14

\begin{abstract}
Objective: To analyze the clinical characteristics of acute cerebrovascular disease.

Methods: 263 patients with acute cerebrovascular disease admitted in our hospital from January 2013 to January 2015 were selected as the study objects and their clinical data were analyzed.

Results: The incidence of acute cerebrovascular disease was higher in males than in females (1.8:1). The morbidity of acute cerebrovascular disease was higher over 60 years old. The incidence time of acute cerebrovascular disease was 6:00-10:00, and hypertension was the primary risk factor of this disease.

Conclusions: The mortality rate of acute cerebrovascular disease was high, and there were many risk factors associated with the disease. The prevention and treatment of acute cerebrovascular disease should be strengthened.
\end{abstract}

Key Words: Acute cerebrovascular disease, Risk factors, Prevention and treatment

Acute cerebrovascular disease is a common medical and frequently-occurring disease of internal medicine. The incidence of acute cerebrovascular disease is rising year by year, and its mortality is high. ${ }^{[1]}$ A total of 263 cases of patients with acute cerebrovascular disease (confirmed by CT) were enrolled in our hospital. The results were reported below.

\section{Materials and methods}

\subsection{General information}

A total of 263 patients with acute cerebrovascular disease admitted in our hospital from January 2013 to January 2015 were enrolled in the study. There were 171 males and 92 females with an average age of 65 years (ranging from 3088 years). 30-39 years in 11 cases, $40-49$ years in 32 cases, $50-59$ years in 61 cases, $60-69$ years in 88 cases, $>70$ years old in 71 cases.

\subsection{Methods}

Clinical data were analyzed.

\section{Results}

\subsection{Types of acute cerebrovascular diseases}

The incidence of acute cerebrovascular diseases included seasonal cerebral hemorrhage in 46 cases, subarachnoid hemorrhage in 10 cases, cerebral thrombosis in 156 cases, cerebral embolism in 26 cases and transient ischemic attack in 25 cases. The incidence of acute cerebrovascular disease was high in the first and the fourth quarter (see Table 1).

\subsection{Circadian rhythm of acute cerebrovascular dis- ease}

The incidence of ischemic cerebrovascular disease was most happened in 6:00-10:00, and the incidence of hemorrhagic

\footnotetext{
*Correspondence: Ronghua Zhao; E-mail: dcc63@ncspress.com; Address: The Third Affiliated Hospital of Inner Mongolia Medical University, Baotou, Inner Mongolia, China.
} 
cerebrovascular disease was most happened in 6:00-10:00, and 18:00-22:00 (see Table 2).

\subsection{Associated diseases}

211 cases of hypertension, 63 cases of diabetes, 41 cases of coronary heart disease, and 14 cases of rheumatic heart disease were found in this study. Onset and clinical manifestations: in 156 cases of cerebral thrombosis, 60 cases were sudden onset, 96 cases were slightly slow; 60 cases were progressive. In 46 cases of cerebral hemorrhage, 39 cases were acute onset, 7 cases were slight slow. 10 cases of subarachnoid hemorrhage were mostly acute onset, 26 cases of cerebral infarction were acute onset, 25 cases of transient ischemic attack were sudden onset. In the hospital, the temperature was examined to be around $39^{\circ} \mathrm{C}-40.1^{\circ} \mathrm{C}$ in
25 cases, and higher than $40.2^{\circ} \mathrm{C}$ in 10 cases. There were 89 cases of headache, 66 cases of nausea, 27 cases of vomiting coffee-colored liquid, 33 cases of dysphoria, 41 cases of coma, 16 cases of different size of the pupils, 8 cases of needle-shaped pupils, 115 cases of blood pressure (systolic blood pressure) of $>180-119 \mathrm{mmHg}$, and 44 cases over 220 $\mathrm{mmHg}$.

Table 1: Cases in different seasons

\begin{tabular}{lll}
\hline Quarter & $\begin{array}{l}\text { Hemorrhagic } \\
\text { cerebrovascular }\end{array}$ & $\begin{array}{l}\text { Ischemic } \\
\text { cerebrovascular }\end{array}$ \\
\hline The first quarter & 18 & 65 \\
The second quarter & 10 & 42 \\
The third quarter & 8 & 35 \\
The fourth quarter & 17 & 68 \\
\hline
\end{tabular}

Table 2: Circadian rhythm of acute cerebrovascular disease

\begin{tabular}{llllllll}
\hline Group & $\mathbf{n}$ & $\mathbf{2 2 : 0 0 - 2 : 0 0}$ & $\mathbf{2 : 0 0 - 6 : 0 0}$ & $\mathbf{6 : 0 0 - 1 0 : 0 0}$ & $\mathbf{1 0 : 0 0 - 1 4 : 0 0}$ & $\mathbf{1 4 : 0 0 - 1 8 : 0 0}$ & $\mathbf{1 8 : 0 0 - 2 2 : 0 0}$ \\
\hline Hemorrhagic group & 53 & 6 & 8 & 19 & 10 & 6 & 4 \\
$\%$ & 100.00 & 11.3 & 15.1 & 35.9 & 18.9 & 11.3 & 7.5 \\
Ischemic group & 210 & 9 & 49 & 93 & 28 & 16 & 15 \\
$\%$ & 100.00 & 4.3 & 23.3 & 44.3 & 13.3 & 7.6 & 7.2 \\
\hline
\end{tabular}

\subsection{Auxiliary examinations}

CT scan showed the location of the disease: basal ganglia in 221 cases, brain stem in 13 cases, cerebral lobes in 21 cases, cerebellum in 8 cases. In cases of cerebral infarction, large area cerebral infarction in 17 cases, lacunar infarction in 45 cases, multifocal cerebral infarction in 121 cases. White blood cell count $>10.0 \times 10^{9}$ in 109 cases, blood glucose $>6.1 \mathrm{mmol} / \mathrm{L}$ in 118 cases, increased fibrinogen in 101 cases. Electrocardiogram examination results: arrhythmia in 50 cases, myocardial ischemia in 101 cases, and dyslipidemia in 120 cases.

\section{Discussion}

\subsection{Age of onset and gender}

The incidence of acute cerebrovascular disease was higher in males than in females (1.8:1), which may be associated with the higher proportion of smoking and drinking in men. Patients with age $<39$ years old accounted for $4.2 \%$, and the oldest was 30 years old. The age of onset of this group was frequently found among 60-year-old patients, up to $60.5 \%$. Therefore, the higher incidence of acute cerebrovascular disease was associated with older age. In that case, old patients were chosen as the key target of prevention and treatment in clinical.

\subsection{Pathogenetic season}

The incidence of acute cerebrovascular disease was obviously seasonal. From this group of cases, the incidence in the first and the fourth quarter was relatively high, while the other two quarters were relatively low, which was consistent with the low temperature in the first and the fourth quarter of this region. The causes of acute cerebrovascular disease in the cold season may be related to air temperature and pressure. Cold irritation increases the release of adrenaline and norepinephrine, causing vasomotor dysfunction, then leading to arteriole spasticity, blood pressure fluctuations and blood pressure surge. ${ }^{[2]}$ Thus, correct understanding of the relationship between the incidence of acute cerebrovascular disease and the season is needed, and timely warming up and combined with other preventive measures in the high incidence season is proved to be an important way to reduce the incidence of acute cerebrovascular disease.

\subsection{Circadian rhythm}

It has been confirmed by many studies that the incidence of cerebral infarction has obvious circadian rhythm. According to the data, 6:00-10:00 was the peak incidence period of ischemic cerebrovascular disease, and 6:00-10:00, 18:00-22:00 were the two peaks of hemorrhagic cerebrovascular disease. Numerous clinical studies have shown that 
the blood pressure of most hypertensive patients and normal people is fluctuating regularly in $24 \mathrm{~h}$ per day. After waking up in the morning, blood pressure rises sharply, and the morning peak of blood pressure is from 6:00 to 10:00. For hypertensive patients, long acting antihypertensive drugs after waking up in the morning can not only lower morning blood pressure but also smoothen blood pressure by $24 \mathrm{~h}$, which can reduce the incidence of cerebral hemorrhage. ${ }^{[3]}$

\subsection{Hypertension}

Hypertension is the primary risk factor for cerebrovascular disease, ${ }^{[4]}$ which accelerates the incidence of cardiovascular and cerebrovascular diseases. The causes are related to the stress of cerebrovascular events, bladder filling, nausea, pain, the location of the lesion and the type of cerebrovascular disease. The main outcome of hypertension is stroke. The occurrence and prognosis of stroke are closely related to the degree and duration of hypertension. Therefore, wellcontrolled blood pressure is very important for reducing the incidence of stroke.

\subsection{Diabetes}

Diabetes is an independent risk factor for cerebrovascular disease. Cerebrovascular disease caused by diabetic is characterized by younger onset, severe illness and high mortality. Hyperglycemia aggravates further damage of brain tissue in patients with cerebrovascular disease, and the extent of hyperglycemia and neurological deficits are significantly related. Control of blood glucose in the normal range can reduce the risk of stroke and improve the prognosis.

\subsection{Heart disease}

Heart disease is recognized as a risk factor for stroke. Ischemic heart disease, valvular heart disease, heart failure, atrial fibrillation, etc., can increase the risk of stroke. Prevention and treatment of various types of heart disease, es-

\section{References}

[1] Xie LW. Analysis of clinical treatment of 150 cases of acute cerebrovascular disease. Health Way. 2015(09): 289.

[2] Wang J. Characteristics of ambulatory blood pressure in patients with essential hypertension combined with cerebral microhaemorrhage. Chinese Journal of Nervous and Mental Diseases. 2015(07): 438. pecially atrial fibrillation, is essential for the prevention of stroke. ${ }^{[5]}$

\subsection{Lipids}

Dyslipidemia is an important risk factor for ischemic stroke. Dyslipidemia can lead to atherosclerosis, increase the incidence of cerebrovascular disease and mortality. Lower cholesterol levels are mainly intervened by lifestyle and the use of statins to prevent the progression of atherosclerotic lesions.

\subsection{Progressive cerebral infarction}

There were 60 cases of progressive stroke $(22.81 \%)$ in this study. The reasons are as follows: (1) infarct expansion and collateral circulation; (2) brain edema; (3) other factors: high temperature, high blood sugar, infection, and electrolyte imbalance. The patient's condition reached its peak for 1-3 days and was stepped up. Therefore, closely observing the change of the condition and prolonging the time window to $9 \mathrm{~h}$ for immediate thrombolytic therapy can improve the condition of the disease as well as the prognosis of the patients.

\section{Conclusions}

The number of stroke risk factors is increasing, so that the incidence of acute cerebrovascular disease, morbidity, mortality increases. Therefore, it is necessary to educate people to change the way of life, and actively control the risk factors associated with the disease, thus preventing the occurrence of acute cerebrovascular disease and improving prognosis.

\section{Conflicts of Interest Disclosure}

The authors have no conflicts of interest related to this article.

[3] Zhao AY. Investigation and analysis of the time and delay factors of patients with acute cerebrovascular disease. Chinese Journal of Practical Nervous Diseases. 2014(04): 127-129.

[4] Wang GB. Analysis of the prevalence and risk factors of cerebral microhemorrhage in patients with different cerebrovascular diseases. Chinese Journal of Nervous and Mental Diseases. 2013(05): 217218.

[5] Li C. Clinical analysis of the factors of acute cerebrovascular disease. Health Care Today. 2016(02): 43-44. 